\title{
On $q$-Deformed Calculus in Quantum Geometry
}

\author{
Olaniyi S. Maliki' ${ }^{1}$ Emmanuel I. Ugwu \\ ${ }^{1}$ Department of Industrial Mathematics and Applied Statistics, Ebonyi State University, Abakaliki, Nigeria \\ ${ }^{2}$ Department of Industrial Physics, Ebonyi State University, Abakaliki, Nigeria \\ Email: somaliki@yahoo.com, ugwuei@yahoo.com
}

Received 21 March 2014; revised 21 April 2014; accepted 28 April 2014

Copyright (C) 2014 by authors and Scientific Research Publishing Inc.

This work is licensed under the Creative Commons Attribution International License (CC BY).

http://creativecommons.org/licenses/by/4.0/

c) (i) Open Access

\begin{abstract}
The relation between noncommutative (or quantum) geometry and the mathematics of spaces is in many ways similar to the relation between quantum physics and classical physics. One moves from the commutative algebra of functions on a space (or a commutative algebra of classical observable in classical physics) to a noncommutative algebra representing a noncommutative space (or a noncommutative algebra of quantum observables in quantum physics). The object of this paper is to study the basic rules governing $q$-calculus as compared with the classical NewtonLeibnitz calculus.
\end{abstract}

\section{Keywords}

Quantum Geometry, $q$-Numbers, $q$-Factorials, $q$-Calculus

\section{Introduction}

There exists an intimate relationship between classical geometry and physics, and to appreciate this we will consider the Einstein field equations (EFE) of special relativity written as:

$$
G_{\mu v}=8 \pi T_{\mu v}
$$

where $G_{\mu v}=R_{\mu \nu}-\frac{1}{2} g_{\mu v} R$ is the Einstein tensor, $R=g^{\mu v} R_{\mu v}$ is the Ricci tensor. $T_{\mu v}$ is the energy-momentum tensor.

For the moment we are only interested in three basic properties of the above equation.

1) The equation (EFE) is a tensor equation. This is necessarily so, since the principle of invariance under coordinate transformations must hold, in other words the equations of physics must look the same in any frame of reference. 
2) We can interpret equation (EFE) more simply as

Tensor representing geometry of space $=$ Tensor representing energy content of space

i.e. it is the presence of matter in space that distorts the neighbouring geometry. Most equations of mathematical physics can be interpreted similarly.

3) The solution to equation (EFE) is a geometrical object, namely a line element given by

$$
d s^{2}=g_{\mu v} d x^{\mu} d x^{v}
$$

where $g_{\mu v}$ is the metric tensor to be solved for in (EFE).

\subsection{Quantum Geometry}

Every geometry is associated with some kind of space. Quantum (or noncommutative) geometry [1] [2] deals with quantum spaces, including the classical concept of space as a very special case. In classical geometry spaces are always regarded as collections of points equipped with the appropriate additional structure (as for example a topological structure given by the collection of open sets, or a smooth structure given by the atlas). In contrast to classical geometry, quantum spaces are not interpretable in this way. In general, quantum spaces have no points at all! They exhibit non-trivial quantum fluctuations' of geometry at all scales.

In generalizing classical geometry to the non-commutative level, there are two important conceptual steps:

1) Translation of geometry into a commutative algebra format;

2) Non-commutative generalizations.

\subsection{Reformulating Basic Geometrical Concepts}

It turns out that the geometrical structure on any given topological space $X$ is always completely expressible in the language of some associated *-algebra [3] [4].

Points

Let $X$ be a compact topological space, and let $A=C(X)$ be the *-algebra of continuous complex-valued functions on $X$. Every element $x \in X$ naturally gives rise to a linear functional $\chi=\chi_{x}: A \rightarrow \mathbb{C}$ defined by

$$
\chi(f)=f(x)
$$

This map is multiplicative in the sense that

$$
\chi(f g)=\chi(f) \chi(g), \quad \forall f, g \in A
$$

Furthermore, $\chi$ is Hermitian in the sense that $\chi\left(f^{*}\right)=\chi(f)^{*}$, it is also non-zero, i.e. $\chi$ is a character on $A$. Conversely, consider an arbitrary character $\chi: A \rightarrow \mathbb{C}$, then it can be shown that there exists a unique point $x \in X$ such that $\chi=\chi_{x}$. In other words, we have a natural bijection between points of $X$ and characters of $A$. It is important to note that this characterization of points also remains valid at the smooth level, in which $X$ could be a compact smooth manifold and the associated *-algebra consists of smooth functions on $X$.

\subsection{The Gelfand-Naimark Theorem}

The algebra $A=C(X)$ of complex-valued functions on a compact topological space $X$, equipped with the maximum norm

$$
|f|=\max _{x \in X}|f(x)|
$$

is a commutative $C^{*}$-algebra [2]. The classical theorem of Gelfand and Naimark characterizes the algebras of the form $A=C(X)$, as commutative unital $C^{*}$-algebras. This means that for every commutative unital $C^{*}$-algebra $A$ there exists (up to homeomerphisms) a unique compact topological space $X$ such that $A \cong C(X)$.

As we have earlier observed, the points of the space $X$ are recovered as characters of the associated algebra $A$. In terms of this identification, the topology on $X$ coincides with the weak ${ }^{*}$-topology, induced from the dual space $A^{*}$, consisting of continuous linear functionals on $A$. It turns out that homomorphisms between $C^{*}$-algebras are automatically continuous, in particular characters are continuous linear functionals. 


\section{The Quantum Plane}

A simple example of quantum geometry is the quantum plane (Figure 1). Usually, a plane is described by two coordinate functions $x, y$. Naturally, the functions $x y$ and $y x$ are the same since it does not matter whether you measure $x$ first and then $y$ or $y$ first and then $x$. This is precisely what is lost in the quantum world.

In the quantum plane we replace the property $x y=y x$ by $x y=q y x$ where $q$ is some parameter. We no longer have points, however we can continue to work algebraically with $x$ and $y$.

Define $[x, y]=x y-y x$, the commutator bracket. Hence $x y=q y x$ can be rewritten as

$$
[x, y]=(q-1) y x
$$

The commutative case is obtained when $q=1$.

\section{1. $q$-Deformed Calculus}

It is interesting to know that one can really do geometry in this setting, where coordinates do not commute. This is the remarkable discovery in recent times. For example, we can follow the approach of Newton-Leibnitz defining differentiation by

$$
f^{\prime}(y)=\left.x^{-1}(f(x+y)-f(y))\right|_{x \rightarrow 0}
$$

But when $x y \neq y x$ and in particular $x y=q y x$ we get instead

$$
\left[f^{\prime}(x)\right]_{q}=\frac{f(x)-f(q x)}{(1-q) x}
$$

Thus for example the derivative of the function $f(x)=x^{n}$ in this non-commutative setting would be

$$
\left[f^{\prime}(x)\right]_{q}=\frac{x^{n}-q^{n} x^{n}}{(1-q) x}=\left(\frac{1-q^{n}}{1-q}\right) x^{n-1}=\left(1+q+q^{2}+\cdots+q^{n-1}\right) x^{n-1}
$$

We observe here that when $q=1$ the derivative of $x^{n}$ for the commutative case is obtained, i.e. $f^{\prime}(x)=n x^{n-1}$.

\subsection{Basic Notions of $q$-Calculus}

The mathematical study of noncommutative geometry is intimately related to the so-called $q$-calculus ( $q$ - numbers, $q$-factorials, $q$-differentials and integrals, basic $q$-hypergeometric functions, and $q$-orthogonal polynomials). Here we give a brief introduction to $q$-numbers and $q$-factorials which will be required in the subsequent sections.

\subsection{1. $q$-Numbers and $q$-Factorials}

For any nonzero complex number $q$, the $q$-number $[a]_{q}, a \in \mathbb{C}$, is defined by

$$
[a]_{q} \equiv[a]:=\frac{q^{a}-q^{-a}}{q-q^{-1}}=\frac{e^{a h}-e^{-a h}}{e^{h}-e^{-h}}=\frac{\sinh a h}{\sinh h}, \quad q=\exp h
$$

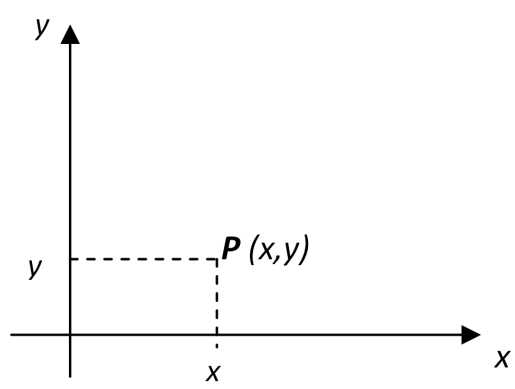

Figure 1 . The quantum plane $(x y \neq y x)$. 
We observe that, $\lim _{q \rightarrow 1}[a]_{q}=a$. The following expression which is easily proved will prove useful.

$$
\llbracket a \rrbracket_{q} \equiv \llbracket a \rrbracket:=\frac{1-q^{a}}{1-q}=1+q+q^{2}+\cdots+q^{a-1}=q^{(a-1) / 2}[a]_{q^{1 / 2}}
$$

Thus given $f(x)=x^{n}$, as shown previously $\left[f^{\prime}(x)\right]_{q}=\llbracket n \rrbracket x^{n-1}$.

\subsubsection{Proposition}

The q-numbers satisfy the following relations derived from the property of the exponential function
1) $[r]=q^{r-1}+q^{r-3}+\cdots+q^{-(r-1)}$
2) $[r+s]=q^{s}[r]+q^{-r}[s]=q^{-s}[r]+q^{r}[s]$
3) $[r-s]=q^{s}[r]-q^{r}[s]=q^{-s}[r]-q^{-r}[s]$
4) $[r][s-t]+[s][t-r]+[t][r-s] \equiv 0$
5) $[r]=[2][r-1]-[r-2]$

The proof of 1) is easy to see from the fact that;

$$
[r]=\frac{q^{r}-q^{-r}}{q-q^{-1}}=\frac{q^{r}\left(1-q^{-2 r}\right)}{q\left(1-q^{-2}\right)}=q^{r-1}\left(\frac{1-\left(q^{-2}\right)^{r}}{1-\left(q^{-2}\right)}\right)
$$

Let $u=q^{-2}, \therefore \frac{1-\left(q^{-2}\right)^{r}}{1-\left(q^{-2}\right)}=\frac{1-u^{r}}{1-u}=1+u+u^{2}+\cdots+u^{r-1}$

Hence;

$$
[r]=q^{r-1}\left(1+q^{-2}+q^{-4}+\cdots+q^{-2(r-1)}\right)=q^{r-1}+q^{r-3}+\cdots+q^{-(r-1)}
$$

The rest of the identities can be proved similarly. It is important to note that the relations 1)-5) remain valid when $q$ is considered an indeterminate. Consequently any $q$-number $[n]_{q}, n \in \mathbb{Z}$, belongs to the space $\mathbb{Z}\left[q, q^{-1}\right]$ of Laurent polynomials [2] in $q$ with integral coefficients.

Suppose $n \in \mathbb{N}$, then we define the $q$-factorial $[n]_{q} ! \equiv[n]$ ! by setting;

$$
[n] !=[1][2] \ldots[n], \quad[0] !:=1
$$

The following expression is quite useful in the theory of hypergeometric functions as well as in combinatorics.

$$
\{a: q\}_{n}:=\prod_{j=1}^{n}\left(1-a q^{j-1}\right)=(1-a)(1-a q)\left(1-a q^{2}\right) \cdots\left(1-a q^{n-1}\right), \quad\{a: q\}_{0}=1 .
$$

It is now possible for us to relate the above with the $q$-factorials. We observe that;

$$
[n] !=\frac{q^{-n(n-1) / 2}}{\left(1-q^{2}\right)^{n}}\left\{q^{2}: q^{2}\right\}_{n}
$$

From Equation (2.3) we note that $\left\{q^{-m}: q\right\}_{n}=0, \forall n=m+1, m+2, \cdots$ For $|q|<1$, define

$$
\{a: q\}_{\infty}:=\lim _{n \rightarrow \infty}\{a: q\}_{n}=\prod_{j=1}^{\infty}\left(1-a q^{j-1}\right)
$$

which converges $\forall a \in \mathbb{C}$, and defines an analytic function on $\mathbb{C}$. 


\subsubsection{Proposition}

$$
\forall a \in \mathbb{C},|q|<1,\{a: q\}_{n}=\frac{\{a: q\}_{\infty}}{\left\{a q^{n}: q\right\}_{\infty}} .
$$

\section{Proof}

$$
\begin{aligned}
\frac{\{a: q\}_{\infty}}{\left\{a q^{n}: q\right\}_{\infty}}=\frac{\prod_{j=1}^{\infty}\left(1-a q^{j-1}\right)}{\prod_{j=1}^{\infty}\left(1-a q^{n+j-1}\right)} & =\frac{(1-a)(1-a q)\left(1-a q^{2}\right)\left(1-a q^{3}\right) \cdots\left(1-a q^{n-1}\right)\left(1-a q^{n}\right)\left(1-a q^{n+1}\right) \cdots}{\left(1-a q^{n}\right)\left(1-a q^{n+1}\right)\left(1-a q^{n+2}\right) \cdots\left(1-a q^{2 n-1}\right) \cdots} \\
& =(1-a)(1-a q)\left(1-a q^{2}\right)\left(1-a q^{3}\right) \cdots\left(1-a q^{n-1}\right)=\{a: q\}_{n}
\end{aligned}
$$

Remark: We can also define and show that;

$$
\{a: q\}_{-n}=\frac{\{a: q\}_{\infty}}{\left\{a q^{-n}: q\right\}_{\infty}}=\frac{1}{\left\{a q^{-n}: q\right\}_{n}}
$$

\subsection{4. $q$-Binomial Coefficients}

The $q$-binomial coefficients are defined by the formula;

$$
\left\{\begin{array}{c}
n \\
m
\end{array}\right\}_{q} \equiv\left\{\begin{array}{c}
n \\
m
\end{array}\right\}:=\frac{\{q: q\}_{n}}{\{q: q\}_{m}\{q: q\}_{n-m}}=\frac{[n]_{q^{1 / 2}} ! q^{(n-m) m / 2}}{[m]_{q^{1 / 2}} ![n-m]_{q^{1 / 2}} !}, \quad \forall n, m \in \mathbb{N} \cup\{0\}, n \geq m .
$$

Remark: There exist a close analogy between the classical binomial coefficients $\left(\begin{array}{c}n \\ m\end{array}\right)=\frac{n !}{(n-m) ! m !}$ and their $q$-analogues. Many of the identities satisfied by the former have their counterparts for the $q$-binomial coefficients. For example the classical identity;

$$
\left(\begin{array}{c}
n+1 \\
m
\end{array}\right)=\left(\begin{array}{c}
n \\
m-1
\end{array}\right)+\left(\begin{array}{l}
n \\
m
\end{array}\right)
$$

simply translates to;

$$
\left\{\begin{array}{c}
n+1 \\
m
\end{array}\right\}_{q}=\left\{\begin{array}{c}
n \\
m-1
\end{array}\right\}_{q}+q^{m}\left\{\begin{array}{c}
n \\
m
\end{array}\right\}_{q}=q^{n-m+1}\left\{\begin{array}{c}
n \\
m-1
\end{array}\right\}_{q}+\left\{\begin{array}{l}
n \\
m
\end{array}\right\}_{q}
$$

\subsubsection{Proposition}

Let $x$ and $y$ be noncommuting variables satisfying the relation $x y=q y x$, then we have

$$
(x+y)^{n}=\sum_{m=0}^{n}\left\{\begin{array}{l}
n \\
m
\end{array}\right\}_{q} y^{m} x^{n-m}=\sum_{m=0}^{n}\left\{\begin{array}{l}
n \\
m
\end{array}\right\}_{q^{-1}} x^{m} y^{n-m}
$$

In case $q$ is a primitive $p^{\text {th }}$ root of unity, and $p$ is odd, then

$$
(x+y)^{p}=x^{p}+y^{p}
$$

\section{Proof}

Equation (2.9) can be established by induction on $n$, and employing the first identity in (2.8). The second assertion follows directly from (2.9). Observe that:

$$
(x+y)^{p}=\sum_{m=0}^{p}\left\{\begin{array}{l}
p \\
m
\end{array}\right\}_{q} y^{m} x^{p-m}=\left\{\begin{array}{l}
p \\
0
\end{array}\right\}_{q} x^{p}+\left\{\begin{array}{l}
p \\
p
\end{array}\right\}_{q} y^{p}+\sum_{m=1}^{p-1}\left\{\begin{array}{l}
p \\
m
\end{array}\right\}_{q} y^{m} x^{p-m}
$$

From (2.7) 


$$
\left\{\begin{array}{l}
p \\
m
\end{array}\right\}_{q}=\frac{\{q: q\}_{p}}{\{q: q\}_{m}\{q: q\}_{p-m}}=\frac{[p]_{q^{1 / 2}} ! q^{(p-m) m / 2}}{[m]_{q^{1 / 2}} ![p-m]_{q^{1 / 2}} !}=0, \quad m=1,2, \ldots, p-1 .
$$

and $\left\{\begin{array}{l}p \\ 0\end{array}\right\}_{q}=\left\{\begin{array}{l}p \\ p\end{array}\right\}_{q}=1$

\section{3. $q$-Differential and $q$-Integral Operators}

The following are important basic notions derived from their analogue in classical calculus, and will be employed subsequently.

\subsection{The $q$-Differential Operator}

For $1 \neq q \in \mathbb{C}$, we define the $q$-differential operator $D_{q}$ by:

$$
D_{q} f(x)=\frac{f(x)-f(q x)}{(1-q) x}
$$

Note that $D_{q} \rightarrow d / d x$ as $q \rightarrow 1$.

\section{Proposition:}

$$
D_{q} f(x)=\sum_{r=0}^{\infty} \frac{(q-1)^{r}}{(r+1) !} x^{r} \frac{d^{r+1}}{d x^{r+1}} f(x), \quad \forall 1 \neq q \in \mathbb{C} .
$$

Provided the expression on the right hand side exists.

\section{Proof}

Let $x+h=q x$, then by Taylor's series, $f(x+h)=f(x)+h f^{\prime}(x)+\frac{h}{2 !} f^{\prime \prime}(x)+\cdots$

$$
\therefore \quad f(x+h)-f(x)=h f^{\prime}(x)+\frac{h}{2 !} f^{\prime \prime}(x)+\cdots=\sum_{r=0}^{\infty} \frac{h^{r+1}}{(r+1) !} \frac{d^{r+1}}{d x^{r+1}} f(x)
$$

Setting $h=(q-1) x$, we have:

$$
D_{q} f(x)=\frac{f(q x)-f(x)}{(q-1) x}=\frac{1}{(q-1) x} \sum_{r=0}^{\infty} \frac{[(q-1) x]^{r+1}}{(r+1) !} \frac{d^{r+1}}{d x^{r+1}} f(x)=\sum_{r=0}^{\infty} \frac{(q-1)^{r} x^{r}}{(r+1) !} \frac{d^{r+1}}{d x^{r+1}} f(x)
$$

The formula for the product of two functions is given by

$$
D_{q}\{u(x) v(x)\}=\frac{u(x) v(x)-u(q x) v(q x)}{x(1-q)}=\frac{v(x)\{u(x)-u(q x)\}+u(q x)\{v(x)-v(q x)\}}{x(1-q)}
$$

We can now define the $q$-analogue of the Newton-Leibnitz rule:

$$
D_{q}\{u(x) v(x)\}=v(x) d_{q} u(x)+u(q x) d_{q} v(x)
$$

Thus,

$$
\begin{aligned}
D_{q}^{2} f(x)=D_{q}\left(D_{q} f(x)\right) & =D_{q}\left(\sum_{r=0}^{\infty} \frac{(q-1)^{r} x^{r}}{(r+1) !} \frac{d^{r+1}}{d x^{r+1}} f(x)\right) \\
& =\sum_{r=0}^{\infty} \frac{(q-1)^{r}}{(r+1) !} D_{q}\left\{x^{r} \frac{d^{r+1}}{d x^{r+1}} f(x)\right\} \\
& =\sum_{r=0}^{\infty} \frac{(q-1)^{r}}{(r+1) !}\left\{\left(\frac{d^{r+1}}{d x^{r+1}} f(x)\right) D_{q} x^{r}+(q x)^{r} D_{q}\left(\frac{d^{r+1}}{d x^{r+1}} f(x)\right)\right\}
\end{aligned}
$$


By induction on $n$, it follows from (3.3) that:

$$
D_{q}^{n} f(x)=\frac{x^{-n} q^{-n(n-1) / 2}}{(q-1)^{n}} \sum_{j=0}^{n}\left\{\begin{array}{l}
n \\
j
\end{array}\right\}_{q}(-1)^{j} q^{j(j-1) / 2} f\left(q^{n-j} x\right)
$$

As a special case when $n=2, D_{q}^{2} f(x)$ is evaluated to give:

$$
D_{q}^{2} f(x)=\frac{1}{q(q-1)^{2} x^{2}}\left\{f\left(q^{2} x\right)-(1+q) f(q x)+q f(x)\right\}
$$

\subsection{The $q$-Integral Operator}

The $q$-integral operator will be defined as the inverse of the $q$-differential operator.

Given $d_{q} F(x)=f(x)$, we have:

$$
F(x)-F(q x)=(1-q) x f(x)
$$

It then follows that; $F\left(q^{j} x\right)-F\left(q^{j+1} x\right)=(1-q) q^{j} x f\left(q^{j} x\right), j=0, \ldots,(n-1)$.

Hence, summing these relations over $j$ gives:

$$
F(x)-F\left(q^{n} x\right)=(1-q) x \sum_{r=0}^{n-1} q^{r} f\left(q^{r} x\right)
$$

Assuming $q \in(0,1)$ so that $F\left(q^{n} x\right) \rightarrow F(0)$ as $n \rightarrow \infty$, it follows that

$$
F(x)-F(0)=(1-q) x \sum_{r=0}^{\infty} q^{r} f\left(q^{r} x\right)
$$

We can now formally define the $q$-integral of a function $f(x)$ on a given interval $[0, \alpha]$ as

$$
\int_{0}^{\alpha} f(x) \mathrm{d} x_{q}=\alpha(1-q) \sum_{r=0}^{\infty} q^{r} f\left(q^{r} \alpha\right)=\sum_{r=0}^{\infty}\left(x_{r}-x_{r+1}\right) f\left(x_{r}\right), \quad x_{r}=\alpha q^{r}
$$

On the semi-infinite interval $[\alpha, \infty)$, the $q$-integral of a function $f(x)$ is defined as:

$$
\int_{\alpha}^{\infty} f(x) \mathrm{d}_{q} x=\alpha(1-q) \sum_{r=1}^{\infty} q^{-r} f\left(q^{-r} \alpha\right)
$$

Over any closed interval $[\alpha, \beta]$, the $q$-integral of a function $f(x)$ is given by

$$
\int_{\alpha}^{\beta} f(x) \mathrm{d}_{q} x=\int_{0}^{\beta} f(x) \mathrm{d}_{q} x-\int_{0}^{\alpha} f(x) \mathrm{d}_{q} x
$$

We now define the integral over the interval $[0, \infty)$. This is achieved by setting $\alpha=1$ in Equations (3.8) and (3.9) and summing to get:

$$
\int_{0}^{\infty} f(x) \mathrm{d}_{q} x=\int_{0}^{1} f(x) \mathrm{d}_{q} x+\int_{1}^{\infty} f(x) \mathrm{d}_{q} x=(1-q) \sum_{r=-\infty}^{\infty} q^{r} f\left(q^{r} \alpha\right)
$$

The integration by parts formula of Newton-Leibnitz calculus is interpreted in the present noncommutative context as:

$$
\int f_{2}(x) D_{q} f_{1}(x) \mathrm{d}_{q} x=f_{1}(x) f_{2}(x)-\int f_{1}(q x) D_{q} f_{2}(x) \mathrm{d}_{q} x
$$

\section{Application}

There are a number of applications of the foregoing, we mention here just two, namely:

1) $q$-binomial formulae in two variables satisfying a quadratic relation, this has recently been published in [5] and [6]. These relations have applications in quantum group theory and non-commutative geometry. 
2) A recent trend in modern physics is the study of the quantum anti-de Sitter space [7] [8] possibly in connection with $q=$ root of unity [9].

\section{Acknowledgements}

This work began at the African Institute for mathematical sciences (AIMS) in Muizenberg South Africa, when the first author visited in 2010. I wish to thank the director Prof Fritz Hahne for giving me a postdoctoral fellowship, and for providing a conducive environment which made this research possible.

\section{References}

[1] Connes, A. (1986) Non-Commutative Differential Geometry. Extrait des Publications Mathematiques-IHES, 62. (cited in: Qauntum Principal Bundles and Their Characteristic Classes (pdf), by MICO DURDEVIC, arXiv:q-alg/960505008vi (5 May 1996))

[2] Connes, A. (1994) Noncommutative Geometry. Academic Press, New York.

[3] Brateli, O. and Robinson, D. (1979) Operator Algebras and Quantum Statistical Mechanics, Volumes 1/2. SpringerVerlag, Berlin.

[4] Brown, L.G., Douglas, R.G. and Filmore, P.G. (1977) Extensions of $C^{*}$-Algebras and K-Homology. Annals of Mathematics, 105, 265-324. http://dx.doi.org/10.2307/1970999

[5] Benaoum, H.B. (1999) ( $q$; h)-Analogue of Newton's Binomial Formula. Journal of Physics A: Mathematical and General, 32, 2037-2040. http://dx.doi.org/10.1088/0305-4470/32/10/019

[6] Rosengren, H. (1999) Multivariable Orthogonal Polynomials as Coupling Coefficients for Lie and Quantum Algebra Representations. Dissertation, Centre for Mathematical Sciences, Mathematics (Faculty of Science), Lund, 167.

[7] Kowalski-Glikman, J. (1998) Black Hole Solution of Quantum Gravity. Physics Letters A, 250, 62-66. http://dx.doi.org/10.1016/S0375-9601(98)00706-3

[8] Chang, Z. (1999) Quantum Anti-De Sitter Space. (reprint)

[9] Steinacker, H. (1998) Finite Dimensional Unitary Representations of Quantum Antide Sitter Groups at Roots of Unity. Communications in Mathematical Physics, 192, 687-706. http://dx.doi.org/10.1007/s002200050315 